\title{
Evaluation of motor complications in Parkinson's disease: understanding the perception gap between patients and physicians
}

Hiromu Ogura

Fukuoka University

Ryoko Nakagawa

AbbVie GK

Miwako Ishido

AbbVie GK

Yoko Yoshinaga

AbbVie GK

Jun Watanabe

AbbVie GK

Kanako Kurihara

Fukuoka University

Yuka Hayashi

Fukuoka University

Koichi Nagaki

Fukuoka University

Takayasu Mishima

Fukuoka University

Shinsuke Fujioka

Fukuoka University

Yoshio Tsuboi ( $\nabla$ tsuboi@cis.fukuoka-u.ac.jp )

Fukuoka University

\section{Research Article}

Keywords: dyskinesia, morning akinesia, observational study, Parkinson's disease, QoL, wearing-off

Posted Date: March 16th, 2021

DOI: https://doi.org/10.21203/rs.3.rs-289875/v1 
License: (c) (i) This work is licensed under a Creative Commons Attribution 4.0 International License. Read Full License

Version of Record: A version of this preprint was published at Parkinson's Disease on December 22nd, 2021. See the published version at https://doi.org/10.1155/2021/1599477. 


\section{Abstract}

Background: Patients with Parkinson's disease (PD) receiving levodopa treatment often report the motor complications including wearing-off (WO), dyskinesia and morning akinesia phenomena. Since motor complications are associated with a decrease in patient quality of life (QoL), it is important to identify its occurrence and commence immediate management. This study investigated whether differences in the perception of motor complications exist between patients and their physicians in routine clinical practice.

Methods: Following an internet-based screening survey, questionnaires were distributed to physicians and their patients in Japan. The 9-item Wearing-off Questionnaire (WOQ-9) assessed the presence of WO objectively; patients with WOQ-9 scores $\geq 2$ were considered to have WO. McNemar's tests were used to compare physician assessment with the WOQ-9, patient self-awareness with physician assessment, and patient self-awareness with the WOQ-9, separately. Morning akinesia, dyskinesia, and QoL were also assessed.

Results: A total of 235 patients with PD and their 92 physicians participated in this survey. A significant discordance was observed between the WOQ-9 and physician assessment of WO $(67.2 \%$ vs $46.0 \%$; $p<0.0001)$. Furthermore, patient self-awareness of WO was $35.3 \%(p=0.0004$, vs physician). Morning akinesia (patient, $58.7 \%$; physician, $48.9 \%$; $p=0.0032$ ), dyskinesia (patient, $34.0 \%$; physician, $23.4 \%$; $p=0.0006$ ), and bodily discomfort (patient, 25.0; physician, $0.0 ; p=0.0102$ ) of $Q$ oL were underrecognized by physicians.

Conclusions: This study investigated differences in the perception of WO between patients with PD and their physicians in routine clinical practice and highlighted that patients have a low awareness of the symptoms of WO compared with physician assessments and the WOQ-9. Conversely, morning akinesia, dyskinesia, and bodily discomfort were underrecognized by physicians.

\section{Background}

Parkinson's disease (PD) is a complex, heterogeneous, neurodegenerative disease [1] characterized by progressive motor symptoms, including tremor, rigidity, and bradykinesia, with postural instability often appearing as the disease progresses [2]. PD is also associated with various nonmotor symptoms, such as cognitive and psychiatric disturbances, autonomic dysfunction, sleep disorders, pain, fatigue, and olfactory dysfunction [1]. Since PD is an incurable progressive condition, the aim of treatment is to control symptoms for as long as possible, improve mobility and function, and maintain overall patient quality of life (QoL) [3].

International guidelines, including those of the Japanese Society of Neurology, recommend either levodopa or a dopamine agonist for the symptomatic treatment of PD $[4,5]$. As the disease progresses, most patients receiving long-term treatment with levodopa will develop motor complications [6, 7]. Frequently, the first motor complication to appear is the "wearing-off" (WO) phenomenon, where the symptomatic benefit of a certain dose of levodopa is not maintained until the next dose; other motor 
complications include dyskinesia in the intermediate stage and complex motor fluctuations in the advanced stage [7]. The occurrence of WO increases gradually, with the majority of patients with PD developing WO within 10 years after initiation of levodopa therapy [8]. WO has also been shown to be common in the early stages of PD. Results from a survey conducted in an Asian population reported that $29 \%$ of patients with levodopa duration of $<1$ year experienced WO, which increased to $68.3 \%$ after $>10$ years of treatment [9]. Since the occurrence of WO is associated with a decrease in patient QoL [8], it is important to identify its occurrence and commence immediate management.

The recognition of WO symptoms, which has typically relied on physician judgment, can be challenging, especially in the early stages of disease, and may therefore be overlooked in routine clinical practice [8, $10,11]$. Results from a survey that explored issues around WO and QoL reported that although the majority of patients ( $87 \%$ ) stated that they understood what "WO" meant, only $30 \%$ of patients provided correct answers on further questioning [12]. Since the early detection of initial motor complications is essential for timely assessment, to optimize therapy and to improve quality of care and patient outcomes, several questionnaires have been developed to improve the recognition of WO. The 32-item Wearing-off Questionnaire (WOQ-32) consists of a checklist of symptoms for patients to check and asks if their symptoms improve with medication [10]. For practical reasons, this questionnaire was adapted to a 19item questionnaire (WOQ-19) and later a 9-item questionnaire (WOQ-9), which had the same features but was more suitable for routine clinical use $[13,14]$.

WO has been shown to be underestimated by physicians. Indeed, results from two trials reported that patients identified the presence of WO by the self-administered WOQ-9 [11] and WOQ-19 [8] more frequently than physicians during neurological evaluation. Although several studies have examined the potential differences in the perception of WO between patients and physicians [8, 10, 14], detailed investigations, particularly in Japan, are lacking. We aimed to explore whether there were any differences in the perception of WO, as well as other health-related outcomes, between patients with PD and their physicians, as assessed in routine clinical practice in Japan.

\section{Methods}

\section{Study design}

This was an observational, cross-sectional study conducted in Japan. This study was compliant with the local laws and regulations and was performed in accordance with the guidelines for Good Pharmacoepidemiology Practices in noninterventional studies and the Japan Ethical Guidelines for Medical and Health Research Involving Human Subjects.

This study was approved by the external institutional review board of the nonprofit organization MINS (Tokyo, Japan. Approval \#180205).

\section{Study methods}


A preliminary internet-based screening survey was first conducted to select physicians who had treated more than five patients per month. The study then consisted of two sequential periods. First, a screening was conducted to determine physician eligibility and interest in participating in this study over the internet. Second, the main survey was conducted, which consisted of instructions for physicians (Supplementary Table 1), patient demographics and disease characteristics, the WOQ-9 for completion by patients, and the 8-item Parkinson's Disease Questionnaire Summary Index (PDQ-8 SI) for completion by both physicians and patients (Supplementary Table 2-4). Data were collected for matched pairs.

Selected physicians received the sealed patient questionnaires, which were given to patients who fulfilled the study inclusion criteria. Physicians did not know the contents of the patient questionnaire. Each physician was required to recruit at least three patients during the study period. Both patients and their physicians were required to complete the questionnaires within 14 days of the clinic appointment. The completed patient and physician questionnaires were collected separately by mail. No specific questions on adverse events were included in the survey.

\section{Eligibility}

Adult patients with a confirmed diagnosis of idiopathic PD who had received any treatment for PD continuously for $>1$ year and were willing and able to provide informed consent were included in the study.

Physicians who had treated at least five patients per month and were willing and able to provide informed consent were also included in the study.

Patients unable to complete the questionnaires by themselves and who had no support for questionnaire completion from caregivers were excluded from the study. In addition, patients and physicians who were unable to submit the questionnaires within 14 days after the clinic appointment were also excluded.

\section{Study endpoints}

The primary endpoint was to compare the proportion of patients identified as having WO by the WOQ-9 and by physician assessment. In our previous study [15], the Japanese version of WOQ-9 with one positive response showed high sensitivity (94.1\%), low specificity (39.2\%), and reasonable accuracy $(70.0 \%)$ for diagnosing WO. With more than two positive responses, the sensitivity of the WOQ-9 decreased from $94.1-87.1 \%$, while specificity increased from $39.2-72 \%$, and accuracy for detecting WO also increased from $70.0-81.4 \%$. Therefore, patients with WOQ-9 scores $\geq 2$ were considered to have WO in this study.

Secondary endpoints included a comparison of the differences between patient self-awareness of WO and physician assessment of WO, an assessment of the differences in the reporting of morning akinesia and dyskinesia between patient self-assessment and physician judgment, and an evaluation of the reported differences in QoL between patient self-assessment and physician judgement, as assessed by the PDQ-8 SI. 


\section{Sample size calculation and statistical analyses}

It was estimated that recruitment of approximately 600 physicians would be feasible. Based on a cooperation rate of $20 \%$, each physician recruited at least three patients. The return rate of matched pairs of the questionnaires was estimated to be $60 \%$. Based on these assumptions, the number of patients required for appropriate statistical power was approximately $210: 600 \times 20 \%$ (cooperation rate) $\times 60 \%$ (paired) $\times 3$ patients (per physician) $=216$. A previous study reported that $15.1 \%$ of patients were identified as having WO by WOQ-9 questionnaire who were not identified by neurologists, and $2.0 \%$ were identified as patients with WO by neurologists who were not identified by WOQ-9 questionnaire even in specialized centers [16]. Assuming discordance rates of $15 \%$ and $2 \%$, a sample size of 76 was considered sufficient to detect differences in the reporting of WO between physicians and patients using the McNemar's test with a type I error rate of $5 \%$ and power of $80 \%$. Therefore, a total of 210 patients and 70 physicians were required for study enrollment. Descriptive statistics were used to describe patient demographic data of all patients who met the inclusion criteria and returned their questionnaires.

The primary endpoint was evaluated using the McNemar's test. In addition, the kappa coefficient and 95\% confidence interval were estimated for the proportion of patients identified with WO. Secondary endpoints that included differences in the reporting of morning akinesia and dyskinesia between patient selfawareness and physician assessment were evaluated using the McNemar's test. The difference in PDQ-8 SI score, as assessed by patients and physicians, was evaluated using $t$ test or the Wilcoxon rank-sum test after determination of data distribution by the Shapiro-Wilk test.

\section{Results}

\section{Patient demographics and baseline clinical characteristics}

Overall, 237 patients with PD and 92 physicians were enrolled in the study from multiple centers across Japan between April 2018 and October 2018. Two patients were excluded as they were not taking any medications for PD; therefore, a total of 235 patients and 92 physicians participated in the study.

Table 1 shows the demographics and clinical characteristics of the patient population. The mean age of patients was 74.0 years, and $51.9 \%$ were women. The average age at PD diagnosis was 65.3 years, and the mean duration of PD was 8.5 years. The majority of patients were in Hoehn and Yahr (H\&Y) stage 3 $(25.5 \%)$ or stage $4(26.8 \%) ; 9.4 \%$ of patients were in H\&Y stage 5.

\section{Table 1}

Patient demographics and baseline clinical characteristics. 


\begin{tabular}{cc}
\hline Characteristic & $\mathrm{N}=235$ \\
\hline Age, mean (SD), years & $74.0(9.4)$ \\
$<65, \mathrm{n}(\%)$ & $31(13.2)$ \\
$\geq 65, \mathrm{n}(\%)$ & $204(86.8)$ \\
Age at PD diagnosis, mean (SD)*, years & $65.3(10.8)$ \\
Duration of PD, mean (SD), years & $8.5(6.2)$ \\
Female, n (\%) & $122(51.9)$ \\
H\&Y stage, n (\%) & \\
1 & $27(11.5)$ \\
2 & $30(12.8)$ \\
3 & $60(25.5)$ \\
4 & $63(26.8)$ \\
5 & $22(9.4)$ \\
Current employment status, n (\%) & $19(8.1)$ \\
Full-time & $10(4.3)$ \\
Part-time & $54(23.0)$ \\
Housekeeping & $152(64.7)$ \\
Not working & $15.8(10.8)$ \\
\hline
\end{tabular}

H\&Y: Hoehn and Yahr; PD: Parkinson's disease; SD: standard deviation. Unknown/missing data are not listed.

*When the age was the same as the age at diagnosis, the age at diagnosis was regarded as missing data.

\section{Presence of WO}

The WOQ-9 identified the signs and symptoms of WO in $67.2 \%$ of cases (Table 2). WO was observed in $46.0 \%$ of cases by physician assessment and in $35.3 \%$ of cases by patient self-awareness (Table 2 ). The discordance between patient self-awareness and physician assessment was observed in 49 cases $(20.9 \% ; p=0.0004) ; 37$ cases $(15.7 \%)$ underrecognized and 12 cases $(5.2 \%)$ overestimated their WO compared with the physician assessment (Table 3). The discordance between patient self-awareness and the WOQ-9 and physician assessment and the WOQ-9 was observed in 99 cases (42.1\%; $p<0.0001$, sum of 12 and 87 cases) and 92 (39.1\%; $p<0.0001$, sum of 21 and 71 cases), respectively (Table 4).

\section{Table 2}

Differences in the perception of WO, morning akinesia, and dyskinesia between patients and their physicians. 


\begin{tabular}{lccccccc}
\hline Total N=235 & \multicolumn{2}{c}{$\begin{array}{c}\text { Patient self- } \\
\text { awareness }\end{array}$} & \multicolumn{2}{c}{$\begin{array}{c}\text { Physician } \\
\text { assessment }\end{array}$} & WOQ-9 & $\begin{array}{c}\text { McNemar's } \\
\text { test }\end{array}$ \\
\hline WO & $\mathrm{n}$ & $\%$ & $\mathrm{n}$ & $\%$ & $\mathrm{n}$ & $\%$ & \\
Morning & 83 & 35.3 & 108 & 46.0 & 158 & 67.2 & $\mathrm{p}<0.0001^{*}$ \\
akinesia & 138 & 58.7 & 115 & 48.9 & $\mathrm{NA}$ & $\mathrm{p}=0.0032^{\#}$ \\
Dyskinesia & 80 & 34.0 & 55 & 23.4 & $\mathrm{NA}$ & $\mathrm{p}=0.0006^{\#}$ \\
\hline
\end{tabular}

NA: not applicable; WO: wearing-off; WOQ-9: 9-item Wearing-off Questionnaire.

Patients with WOQ-9 scores $\geq 2$ were regarded as having WO.

*Physician assessment versus WOQ-9.

\# Patient self-awareness versus physician assessment.

\section{Table 3}

Differences in the perception of WO, morning akinesia, and dyskinesia between patient selfawareness and physician assessment

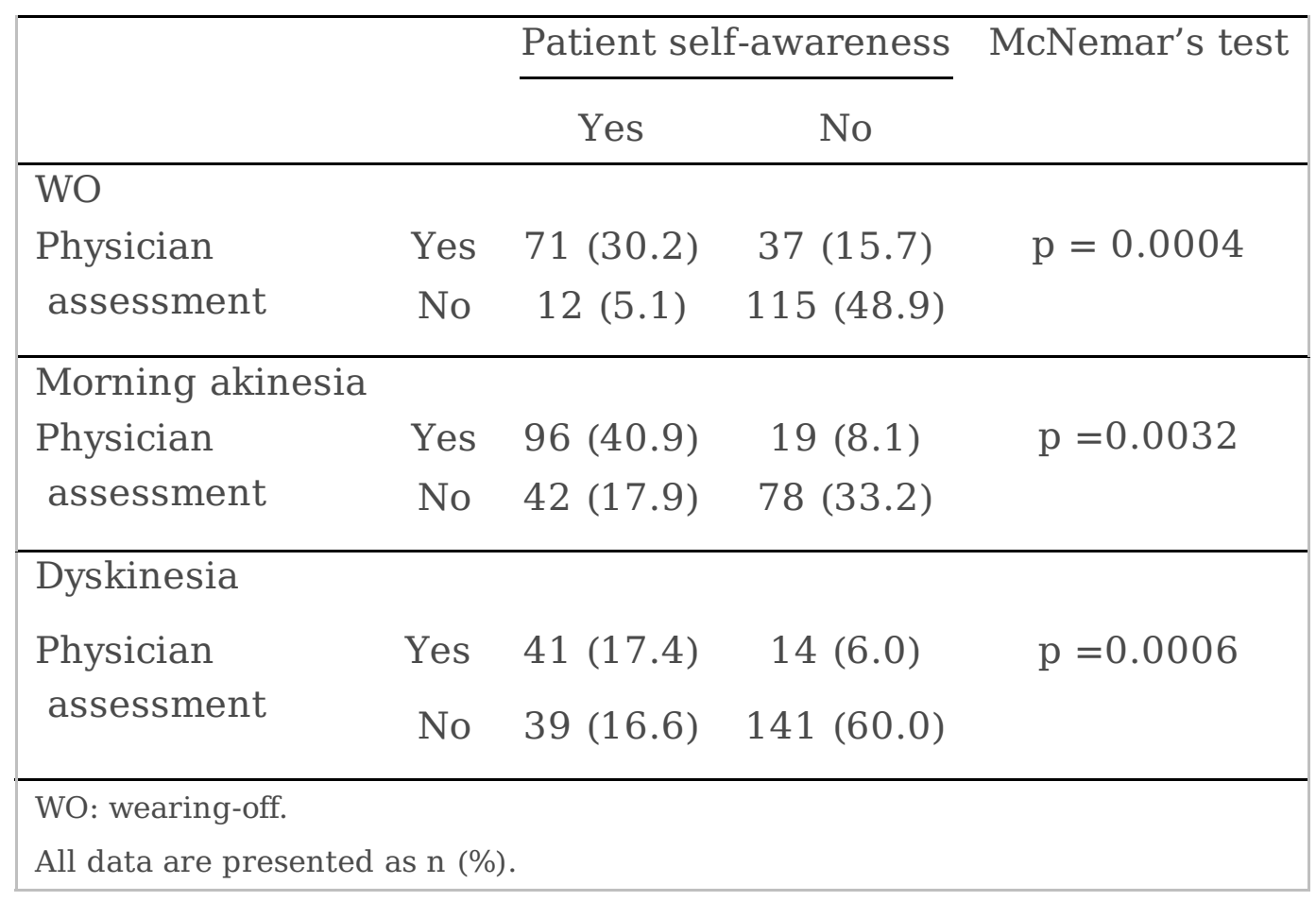

\section{Table 4}

Differences in the assessment of WO between patient self-awareness and physician assessment based on patient's WOQ-9 


\begin{tabular}{|c|c|c|c|c|}
\hline & & Patient & WOQ-9 & McNemar's test \\
\hline & & Yes & No & \\
\hline Patient self-awareness & Yes & $71(30.2)$ & $12(5.1)$ & $p<0.0001$ \\
\hline & No & $87(37.0)$ & $65(27.7)$ & \\
\hline Physician assessment & Yes & $87(37.0)$ & $21(8.9)$ & $\mathrm{p}<0.0001$ \\
\hline & No & $71(30.2)$ & $56(23.8)$ & \\
\hline $\begin{array}{l}\text { WO: wearing-off; WOQ-9: 9-it } \\
\text { All data are presented as n }\end{array}$ & $\mathrm{m} \mathrm{We}$ & ing-off Ques & ionnaire. & \\
\hline Patients with WOQ-9 scores & we & egarded a & aving WO. & \\
\hline
\end{tabular}

\section{Presence of morning akinesia}

Morning akinesia was observed in 115 patients (48.9\%) by physician assessment and in 138 patients $(58.7 \%)$ by patient self-awareness ( $p=0.0032$; Table 2$)$. The presence of morning akinesia was underrecognized by physicians in 42 cases (17.9\%), while by patients in only 19 cases (8.1\%) (Table 3).

\section{Presence of dyskinesia}

Differences in the presence of dyskinesia were observed between physician assessment $(n=55,23.4 \%)$ and patient self-awareness $(n=80,34.0 \%$; $p=0.0006$; Table 2). The presence of dyskinesia was underreported by physicians in 39 patients (16.6\%); in contrast, only 14 patients (6.0\%) were not selfaware of their dyskinesia (Table 3).

\section{QoL assessed by patients and physicians (PDQ-8 SI)}

No significant differences were observed between physician and patient assessment of QoL with the exception of bodily discomfort, which was poorly recognized by physicians (patient median, 25.0 vs physician median 0.0; $p=0.0102$, Table 5). The PDQ-8 SI score as assessed by patients who did not consider the presence of WO by patients, physician, or WOQ-9 was 25.6; that score as assessed by physicians was 28.5 (Supplementary Table 5). On the other hand, even when patients did not consider themselves experiencing WO, the population whom both physicians and WOQ-9 assessed as having WO shows worsened QoL (PDQ8 SI scores are 37.7 and 39.4 assessed by patients and physicians, respectively).

The population in whom patients themselves were aware of morning akinesia, but physicians did not assess the presence of morning akinesia, had worsened QoL (39.9 and 43.2) compared with the population whom both patients and physicians considered the absence of morning akinesia (23.5 and 20.7 assessed by patients and physicians, respectively. Supplementary Table 6).

\section{Table 5}

Quality of life as assessed by patients and physicians (PDQ-8). 


\begin{tabular}{lccccc}
\hline & \multicolumn{2}{c}{ Patient } & Physician & p value \\
\cline { 2 - 5 } & Mean (SD) & $\begin{array}{c}\text { Median } \\
(\text { min, max })\end{array}$ & Mean (SD) & $\begin{array}{c}\text { Median } \\
(\text { min, max })\end{array}$ & \\
\hline ty & $60.5(35.5)$ & $75.0(0,100)$ & $62.1(33.5)$ & $75.0(0,100)$ & 0.5812 \\
ies of daily living & $54.4(36.5)$ & $50.0(0,100)$ & $55.4(35.2)$ & $50.0(0,100)$ & 0.4868 \\
onal well-being & $37.0(31.4)$ & $25.0(0,100)$ & $35.5(30.2)$ & $25.0(0,100)$ & 0.5284 \\
support & $23.6(30.8)$ & $0.0(0,100)$ & $25.7(29.6)$ & $25.0(0,100)$ & 0.3386 \\
tion & $33.5(31.8)$ & $25.0(0,100)$ & $31.7(30.8)$ & $25.0(0,100)$ & 0.4577 \\
tunication & $33.5(32.9)$ & $25.0(0,100)$ & $30.6(31.5)$ & $25.0(0,100)$ & 0.1115 \\
discomfort & $25.1(29.8)$ & $25.0(0,100)$ & $20.1(28.4)$ & $0.0(0,100)$ & 0.0102 \\
a & $28.4(30.8)$ & $25.0(0,100)$ & $30.2(29.0)$ & $25.0(0,100)$ & 0.3281 \\
ary index & $36.9(24.5)$ & $34.4(0,100)$ & $36.4(24.3)$ & $34.4(0,100)$ & 0.7532 \\
\hline
\end{tabular}

PDQ-8; 8-item Parkinson's Disease Questionnaire; SD; standard deviation.

\section{Discussion}

Although levodopa is still recognized as the most effective medication for PD, long-term treatment is often associated with motor complications [17], which impair daily living and have a negative impact on a patient's QoL. Since WO is generally the first motor complication to develop, its early identification is of great importance for the timely optimization of therapy [8].

The overall prevalence rate of WO in this study was $35.3 \%$ (Table 2), which was nearly identical to that reported in another Japanese survey in 407 patients from the Japan Parkinson Disease Association, where $36 \%$ of patients, with a mean age of 69 years and disease duration ranging from 3-9 years, reported WO [18]. Results from this study highlighted the low awareness of WO among patients compared with both the physician assessment and the WOQ-9, which suggests that patients do not easily recognize the early signs of WO. In line with previous reports [10,11], our study confirmed that differences in the perception of WO exist between patients and their physicians, as evidenced by the fact that WO was observed in $46.0 \%$ of patients by physician assessment and in $35.3 \%$ of patients by patient selfawareness. On the other hand, WOQ-9 could detect WO with high sensitivity. The WOQ-9 identified WO in $67.2 \%$ of patients compared with the physician assessment that identified WO in only $46.0 \%$ of patients (Table 2). We considered that patients with WOQ-9 scores $\geq 2$ were having WO according to our previous study [15], which showed that sensitivity and specificity for WOQ-9 scores $\geq 2$ were $87.1 \%$ and $72.2 \%$, respectively, while those for WOQ-9 scores $\geq 1$ were $94.1 \%$ and $39.2 \%$, respectively. Therefore, WOQ-9 scores $\geq 2$ might help the avoiding false positive and maintain high sensitivity. These findings may be attributable to the clear and concise nature of the WOQ-9, which makes it quick and simple to use. In contrast, busy physicians may not always have sufficient time to adequately ask their patients about potential symptoms of WO [10]. Therefore, the WOQ-9 can be an effective screening tool that aids in the diagnosis of WO in patients with PD. 
Morning akinesia, which is common in patients with PD, not only causes significant disability but also has a negative impact on a patient's QoL $[19,20]$. Indeed, results from a European, multicenter, observational study reported that up to $60 \%$ of patients experience morning akinesia, which prevents them from performing morning routines [20]. In our study, patients recognized morning akinesia to a greater extent than physicians, with $58.7 \%$ of patients reporting morning akinesia compared with $48.9 \%$ of physicians (Table 2). This finding is in line with the results of an Italian survey in 151 consecutive patients, which reported that $64.2 \%$ of patients were able to recognize morning akinesia [21]. Taken together, these findings suggest that patients easily recognize the presence of morning akinesia and are therefore more likely to discuss any concerns with their physicians [19]. Moreover, results from our study showed that patients were far more likely to recognize morning akinesia than WO. In contrast, physicians reported similar rates of akinesia and WO (48.9\% and $46.0 \%$, respectively). Consequently, there may be a perception among some physicians that patients are able to identify the early symptoms of WO as easily as those of morning akinesia; however, due to the heterogeneity of the signs and symptoms of WO, patients frequently underrecognize WO, a finding that was observed in our study [10].

Episodes of dyskinesia are a major challenge in the long-term management of patients with PD [22]. It is therefore unsurprising that troublesome dyskinesia was reported by $34.0 \%$ of patients, an observation that corroborates findings from previous studies where treatment-induced dyskinesia occurred in 28.0$40.0 \%$ of patients $[6,22]$. Dyskinesia, as well as morning akinesia, were underreported by a greater number of physicians compared with patients, highlighting the need for careful and accurate sharing of information between patients and their physicians.

Since PD significantly impacts health-related QoL, this study used the PDQ-8 to assess health-related QoL. As expected, PD had a negative impact on patient QoL, which was, in general, consistently reported by both patients and physicians. Interestingly, bodily discomfort was poorly recognized by physicians, a finding that may be attributable to a number of reasons, including the patient's disease profile, pain threshold, the part of the body examined, and the timing of assessment in relation to drug administration $[23,24]$.

The results from this study showed worsened QoL among patients for which both physician and WOQ-9 assessments were positive but patient self-assessments were negative compared with patients for which physician and WOQ-9 assessments and patient self-assessments all were negative (Supplemental Table 5). These data support the hypothesis that patients often underestimate their WO, and therefore, which worsened QoL without awareness. Regarding morning akinesia, worsened QoL was observed among patients for which patient self-awareness was positive but physician assessment was negative compared with those for which both patient self-awareness and physician assessment were negative (Supplemental Table 6). Interestingly, PDQ-8 SI assessed by patients and physicians showed similar scores, which suggests that physicians realized the deteriorated QoL of patients, but did not consider their morning akinesia as the reason of it. 
There are some limitations to our study. First, there are limitations inherent to these types of surveys, and some patients may have been unable to complete the questionnaires because of their physical restrictions. In addition, this survey may have been susceptible to biases such as responder bias, recall bias, and interviewer bias. Along with the well-known limitations of noninterventional and cross-sectional studies, the sample size was also relatively small. Finally, as the focus of the study was on Japan, the generalizability of the results is unclear. However, despite these limitations, results from this study emphasize the importance of using effective screening tools, such as the WOQ-9, to aid physicians in the diagnosis of WO. Moreover, these findings highlight the need for an open dialogue and effective communication and collaboration between patients and physicians. In addition, recent advances in digital technology and biotechnology have led to the development of many types of wearable sensor systems, enabling the continuous long-term monitoring of motor complications $[25,26]$. These sensors, which are unobtrusive and accurate, should further assist physicians in diagnosing and managing the symptoms of WO.

\section{Conclusions}

This study, which investigated differences in the perception of WO between patients with PD and their physicians in routine clinical practice, highlighted that patients have a low awareness of the symptoms of WO compared with physician assessment and the WOQ-9. Conversely, morning akinesia, dyskinesia, and bodily discomfort were underrecognized by physicians.

The use of an objective measure to evaluate WO, such as the WOQ-9, combined with improved patient education and awareness of PD treatments and their associated complications, is of paramount importance for effective patient-physician communication and ultimately to enhance patient care and treatment outcomes.

\section{Abbreviations}

H\&Y: Hoehn and Yahr; PD: Parkinson's disease; PDQ-8: 8-item Parkinson's Disease Questionnaire; PDQ-8 SI: 8-item Parkinson's Disease Questionnaire Summary Index; QOL: quality of life; SD: standard deviation; WO: wearing-off; WOQ-9: 9-item wearing-off questionnaire; WOQ-19: 19-item wearing-off questionnaire; WOQ-32: 32-item wearing-off questionnaire

\section{Declarations}

\section{Acknowledgments}

We sincerely thank all the physicians and patients that cooperated with this study. Medical writing support was provided by Urvashi Nikte, MDS, of Cactus Life Sciences (part of Cactus Communications; Mumbai, India), and was funded by AbbVie GK.

\section{Author contributions}


Study Concept and design of the study: MI, YY, YT. Data Acquisition and analysis: HO, RN, MI, YY, JW, KK, YH, KN, TM, SF, YT. Manuscript Drafting: MI, JW. All authors critically reviewed the manuscript and agreed on this final version to be submitted to the journal.

\section{Funding}

This work was funded by AbbVie GK. AbbVie participated in the study design; research; data collection; data analysis and interpretation; and writing, review, decision to submit for publication, and approval of the manuscript. No honoraria or payments were made for authorship.

\section{Availability of data and materials}

The datasets generated and/or analyzed during the current study are not publicly available due sponsor's policy but are available from the corresponding author on reasonable request.

\section{Ethics approval and consent to participate}

This study was approved by the external institutional review board of the nonprofit organization MINS (Tokyo, Japan. Approval \#180205). Written informed consent was obtained from all physicians and patients participating in the study.

\section{Consent for publication}

Not Applicable.

\section{Competing interests}

Y. Tsuboi has served as an advisor for AbbVie GK and has received research support from the Japan Agency for Medical Research and Development and Kyowa Kirin. R. Nakagawa and J. Watanabe are employees of AbbVie GK and may receive stock or stock options. M. Ishido and Y. Yoshinaga are former AbbVie employees. H. Ogura, K. Kurihara, Y. Hayashi, K. Nagaki, T. Mishima and S. Fujioka report no conflicts of interest.

\section{References}

1. Kalia LV, Lang AE. Parkinson's disease. Lancet. 2015;386:896-912.

2. Goetz CG. The history of Parkinson's disease: early clinical descriptions and neurological therapies. Cold Spring Harb Perspect Med. 2011;1:a008862.

3. Osaki Y, Morita Y, Kuwahara T, Miyano I, Doi Y. Prevalence of Parkinson's disease and atypical parkinsonian syndromes in a rural Japanese district. Acta Neurol Scand. 2011;124:182-7.

4. Fox SH, Katzenschlager R, Lim SY, Barton B, de Bie RMA, Seppi K, et al. International Parkinson and movement disorder society evidence-based medicine review: Update on treatments for the motor symptoms of Parkinson's disease. Mov Disord. 2018;33:1248-66. 
5. Parkinson's disease clinical practice guidelines 2018. https://www.neurologyjp.org/guidelinem/parkinson_2018.html Accessed December 25, 2020.

6. Ahlskog JE, Muenter MD. Frequency of levodopa-related dyskinesias and motor fluctuations as estimated from the cumulative literature. Mov Disord. 2001;16:448-58.

7. Bhidayasiri R, Truong DD. Motor complications in Parkinson disease: clinical manifestations and management. J Neurol Sci. 2008;266:204-15.

8. Stocchi F, Antonini A, Barone P, Tinazzi M, Zappia M, Onofrj M, et al. Early DEtection of wEaring off in Parkinson disease: the DEEP study. Parkinsonism Relat Disord. 2014;20:204-11.

9. Chen W, Xiao Q, Shao M, Feng T, Liu WG, Luo XG, et al. Prevalence of wearing-off and dyskinesia among the patients with Parkinson's disease on levodopa therapy: a multi-center registry survey in mainland China. Transl Neurodegener. 2014;3:26.

10. Stacy M, Bowron A, Guttman M, Hauser R, Hughes K, Larsen JP, et al. Identification of motor and nonmotor wearing-off in Parkinson's disease: comparison of a patient questionnaire versus a clinician assessment. Mov Disord. 2005;20:726-33.

11. Bares M, Rektorova I, Jech R, Farnikova K, Roth J, Ruzicka E, et al. Does WOQ-9 help to recognize symptoms of non-motor wearing-off in Parkinson's disease? J Neural Transm (Vienna). 2012;119:373-80.

12. Matthews H, Stamford J, Saha R, Martin A. Exploring Issues Around Wearing-off and Quality of Life: The OFF-PARK Survey of People with Parkinson's Disease and their Care Partners. J Parkinsons Dis. 2015;5:533-9.

13. Stacy M, Hauser R. Development of a Patient Questionnaire to facilitate recognition of motor and non-motor wearing-off in Parkinson's disease. J Neural Transm (Vienna). 2007;114:211-7.

14. Stacy MA, Murphy JM, Greeley DR, Stewart RM, Murck H, Meng X. The sensitivity and specificity of the 9-item Wearing-off Questionnaire. Parkinsonism Relat Disord. 2008;14:205-12.

15. Fukae J, Higuchi MA, Yanamoto S, Fukuhara K, Tsugawa J, Ouma S, et al. Utility of the Japanese version of the 9-item Wearing-off Questionnaire. Clin Neurol Neurosurg. 2015;134:110-5.

16. Bares M, Rektorova I, Jech R, Farnikova K, Roth J, Ruzicka E, et al. Does WOQ-9 help to recognize symptoms of non-motor wearing-off in Parkinson's disease? J Neural Transm (Vienna). 2012;119:373-80.

17. Jankovic J, Aguilar LG. Current approaches to the treatment of Parkinson's disease. Neuropsychiatr Dis Treat. 2008;4:743-57.

18. Hattori N, Fujimoto K, Kondo T, Murata M, Stacy M. Patient perspectives on Parkinson's disease therapy in Japan and the United States: results of two patient surveys. Patient Relat Outcome Meas. 2012;3:31-8.

19. Chapuis S, Ouchchane L, Metz O, Gerbaud L, Durif F. Impact of the motor complications of Parkinson's disease on the quality of life. Mov Disord. 2005;20:224-30. 
20. Rizos A, Martinez-Martin P, Odin P, Antonini A, Kessel B, Kozul TK, et al. Characterizing motor and non-motor aspects of early-morning off periods in Parkinson's disease: an international multicenter study. Parkinsonism Relat Disord. 2014;20:1231-5.

21. Stocchi F, Coletti C, Bonassi S, Radicati FG, Vacca L. Early-morning OFF and levodopa dose failures in patients with Parkinson's disease attending a routine clinical appointment using Time-to-ON Questionnaire. Eur J Neurol. 2019;26:821-6.

22. Schrag A, Quinn N. Dyskinesias and motor fluctuations in Parkinson's disease. A community-based study. Brain. 2000;123 (Pt 11):2297-305.

23. Brefel-Courbon C, Payoux P, Thalamas C, Ory F, Quelven I, Chollet F, et al. Effect of levodopa on pain threshold in Parkinson's disease: a clinical and positron emission tomography study. Mov Disord. 2005;20:1557-63.

24. Djaldetti R, Shifrin A, Rogowski Z, Sprecher E, Melamed E, Yarnitsky D. Quantitative measurement of pain sensation in patients with Parkinson disease. Neurology. 2004;62:2171-5.

25. Ossig C, Antonini A, Buhmann C, Classen J, Csoti I, Falkenburger B, et al. Wearable sensor-based objective assessment of motor symptoms in Parkinson's disease. J Neural Transm (Vienna). 2016;123:57-64.

26. Rovini E, Maremmani C, Cavallo F. How Wearable Sensors Can Support Parkinson's Disease Diagnosis and Treatment: A Systematic Review. Front Neurosci. 2017;11:555.

\section{Supplementary Files}

This is a list of supplementary files associated with this preprint. Click to download.

- SUPPLEMENTALMATERIAL.pdf 